\title{
PHYSICAL CLASSIFICATION OF SHORT-PERIOD VARIABLES
}

\author{
D. H. P. JONES \\ Royal Greenwich Observatory
}

It is widely accepted that pulsating variables with periods less than 0.2 day fall into two distinct groups. These defined by Kukarkin et al. (1969) as, firstly,

RRs:RR Lyrae type variables with the period not exceeding 0 21 (dwarf Cepheids). Belong to the population of the disk, are absent in clusters. Their luminosity is $2^{m}-3^{m}$ fainter than the luminosity of RRab and RRc stars. A typical representative - SX Phe.

and secondly,

$\delta$ Sct $: \delta$ Scuti type stars. Pulsating variables of spectral class $A$ (late sub-classes) and $F$, the amplitudes of light variation do not exceed, as a rule, $0 m^{m} 1$ (rarely up to $0{ }^{m} 3$ ). The form of light curve strongly varies usually. According to many characteristics resemble dwarf Cepheids, but differ from them by the small amplitudes. Are met in the Hyades-type clusters. Similar to the RRs type stars their periods do not exceed 0.2 . A typical representative $-\delta$ Sct.

However it has been doubted (e.g. Eggen, 1970) whether these variables form two distinct groups or whether they form the ends of a continuum with no well-defined boundary between them. Eggen showed that both groups were represented in both young and old disk populations. Now that homogeneous intermediate band colours are available for a large sample of these variables (Jones, 1971, 1973, and unpublished) it is appropriate to examine whether the colours reveal any clear cut difference. Certainly there is no division by period which for the $\delta$ Sct variables lies between 0.193 $(\delta \mathrm{Sct})$ and 0.055 (HD 100363) and for the RRs variables which lie between 0.178 (VZ Cnc) and 0.055 (SX Phe). Effective temperature can be inferred from the $\beta_{1}$ index of Jones (1973): for the $\delta$ Sct variables it ranges from $7800 \mathrm{~K}$ (HD 170625) to $6800 \mathrm{~K}$ ( $\delta$ Sct, X Cae) and for the RRs from $7800 \mathrm{~K}$ (SX Phe) to $7000 \mathrm{~K}$ (V703 Sco). Metal abundance can be inferred from the $(k-b)_{2}$ index: for the $\delta \mathrm{Sct},[\mathrm{Fe} / \mathrm{H}]$ ranges from +0.4 ( $\zeta$ Pup) to -1.1 (HD 6870) and for the RRs it varies from +0.3 (VZ Cnc) to -0.5 (SX Phe). Again there is no suggestion of a division. The apparent gravities can be inferred from the Balmer jump index $(u-b)_{3}$. Like the other indices this was measured at the instant where $y=\langle y\rangle$ on the falling branch. At this instant the atmosphere is out of hydrostatic equilibrium and a correction must be made for dynamical acceleration. A correction of

$$
1.09 \times 10^{-2}\left(A_{v} / P\right)
$$

in solar units was applied to the gravities. This is based on Bessell's (1969) velocity curve for AI Vel and it assumes that the velocity amplitude is proportional to the visual light amplitude $A_{v}$ (in magnitudes) and that the velocity curves of all variables are similar and independent of the period $P$ (in days). In Figure 1 the gravities so determined are compared with the periods. A partial separation is evident and the 


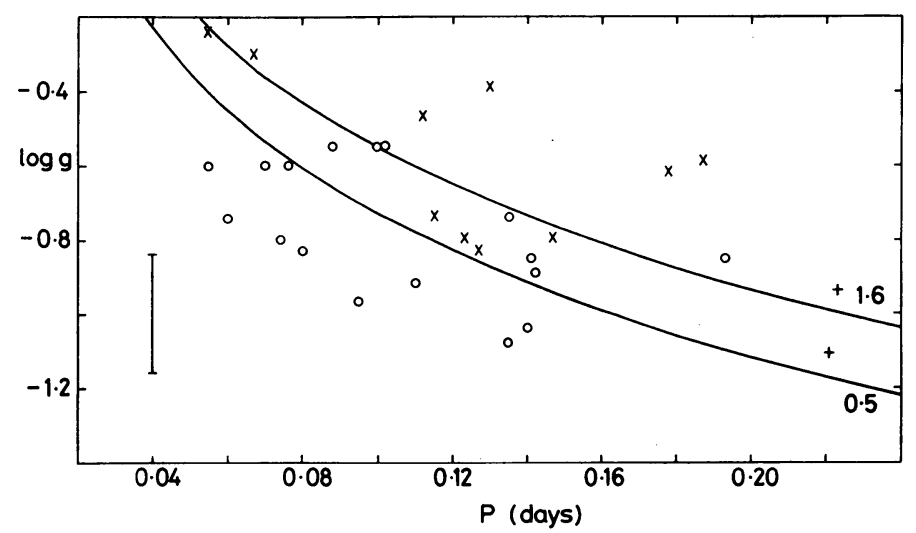

Fig. 1. Plot of $\log$ (gravity in solar units) against period in days. The vertical line is two standard errors in length. $\delta$ Sct variables are circles and RRs diagonal crosses. The plus signs are two obscure variables VX Hya and V753 Cen which may not be related to the others. The curved lines are the period-gravity relations of Otzen Petersen and Jørgensen (1972) for 0.5 and 1.6 solar masses.

errors of observation are so large that the two groups may well be completely separated in reality.

The pulsation equation is (Ledoux and Walraven, 1958; Kinman, 1959)

$$
P \sqrt{ } \rho=Q,
$$

where $P$ is the period (here is days), $\rho$ the central density (here in solar units) and $Q$ is constant for a homologous series of models. This is equivalent to the relation

$$
P \sim g^{-0.75} \mathfrak{M}^{0.25}
$$

where $g$ and $\mathfrak{M}$ are the gravity and mass in solar units. In fact a real series of variable stars will not be homologous, neither will a realistic series of models. Christy (1966) found for his RR lyrae models

$$
P \sim g^{-0.875} \mathfrak{M}^{0.125}
$$

while van Albada and Baker (1971) found, for their own models of RR Lyraes, the relation

$$
P \sim g^{-0.84} \mathfrak{M}^{0.16}
$$

ignoring a slight dependence on temperature. The pulsation of hydrogen-burning models more appropriate to the short period variables has been investigated by Otzen Petersen and Jorgensen (1972). The following formula fits their calculations

$$
\log P=-1.49-0.78 \log g+0.27 \log \mathfrak{M}
$$

with discrepancies $<0.01$ for nearly all models and no significant dependence on temperature. Otzen Petersen and Jørgensen's models range from 0.5 to 1.6 solar masses and the corresponding curves from (6) are drawn in Figure 1. The immediate implication is that the RRs variables are more massive than the $\delta$ Scutis. This is in 
contradiction to Bessell's (1969) suggestion that the RRs variables (AI Velorum stars in his terminology) are in the post-red giant phase of their evolution while the $\delta$ Sct variables are in the pre-red giant phase. Moreover Bessell's result is in accord with Kippenhahn's (1965) theoretical finding that normal main sequence A and F stars are nearly stable against oscillation but that instability increases with decreasing mass. Not only is the present mass difference in the unexpected sense, it is also embarrassingly large; $\Delta \log \mathfrak{M}=0.74 \pm 0.22$ (s.e.). The residual scatter within the two groups is almost entirely ascribable to errors in the measurement of $\log g$. Now there may be difficulties with the models for their first overtone: fundamental period ratios lead to compositions quite different from those derived from the colours by Jones (1973). However the close similarity of Equations (3)-(6) suggests that no feasible change in the models will destroy the weak but positive dependence of period on mass. There is one simple explanation which nearly removes the discrepancy - to postulate that the $\delta$ Sct stars pulsate in the first overtone while the RRs variables pulsate in the fundamental. The corresponding $\Delta \log P=0.12$ and $\Delta \log \mathfrak{M}=0.44$. This suggestion is not inconsistent with the directly determined $Q$ values of Otzen Petersen and Jørgensen (1972) even considering only their well-determined values. It is suggested that the $\delta$ Sct and RRs variables are fundamentally similar groups; hydrogen shell burning stars of about two solar masses. The only difference is the mode of pulsation. What physical difference induces the different modes is not known.

Further evidence for this suggestion is provided by the luminosities. Re-arranging Equation (6) we find

$$
\log P+0.51 \log g+1.08 \log T_{\mathrm{e}}+1.49=0.27 \log L
$$

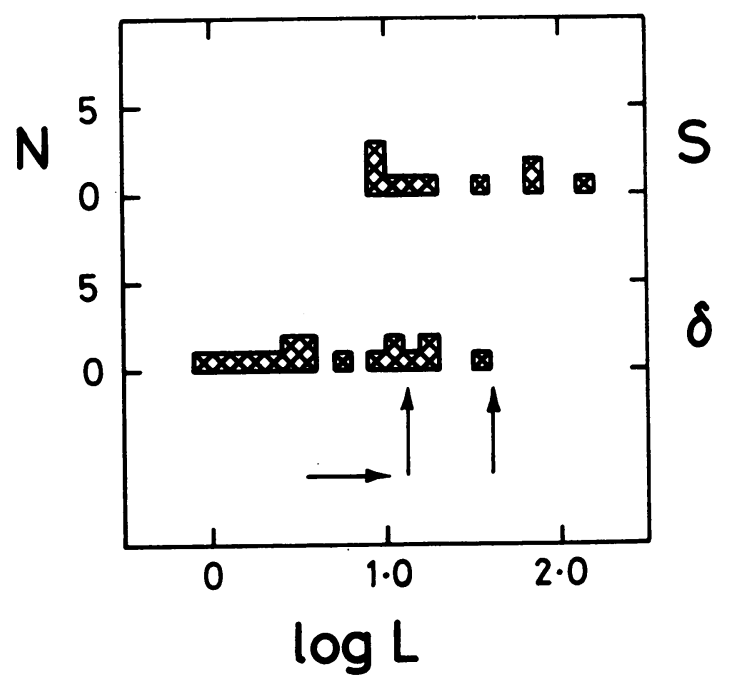

Fig. 2. Histogram of $\log$ (luminosity in solar units) derived from Equation (7) for RRs variables $\mathrm{S}$ and $\delta$ Sct variables $\delta$. The vertical arrows are the preferred luminosities $M_{V}=0.6$ or 1.9 of Eggen (1970). The horizontal arrow is the increase in luminosity if the observed period is the first overtone. 
$T_{\mathrm{e}}$ the effective temperature is inferred from the $\beta_{1}$ index. The histogram of the luminosities so derived is plotted in Figure 2. The two luminosities favoured by Eggen (1970) for variables of both types are marked by vertical arrows. The theoretical luminosities of the RRs variables are in good accord with the observed values but the $\delta$ Sct variables are too faint. The horizontal arrow marks the increase in luminosity a variable receives if its observed period is really the first overtone. This is in the right sense and roughly the right amount to reconcile the luminosities.

\section{References}

Albada, T. S. van and Baker, N.: 1971, Astrophys. J. 169, 311.

Bessell, M. S.: 1969, Astrophys. J. Suppl. 18, 195.

Christy, R. F.: 1966, Ann. Rev. Astron. Astrophys. 4, 353.

Eggen, O. J.: 1970, Publ. Astron. Soc. Pacific 82, 274.

Jones, D. H. P.: 1971, Monthly Notices Roy. Astron. Soc. 154, 79.

Jones, D. H. P.: 1973, Astrophys. J. Suppl. 25, 487.

Kinman, T. D.: 1959, Monthly Notices Roy. Astron. Soc. 119, 134.

Kippenhahn, R.: 1965, Klein. Veröf. Bamberg 4, 40, 7.

Kukarkin, B. V., Kholopov, P. N., Efremov, Yu. N., Kukarkina, N. P., Kurochkin, N. E., Medvedeva, G. I., Perova, N. B., Fedorovich, V. P., and Frolov, M. S.: 1969, Gen. Cat. Var. Stars, Astron. Council of Acad. Sci. USSR, Moscow.

Ledoux, P. and Walraven, Th.: 1958, in S. Flügge (ed.), Handbuch der Physik LI, 431, SpringerVerlag, Berlin.

Otzen Petersen, J. and Jørgensen, H. E.: 1972, Astron. Astrophys. 17, 367. 